\title{
Beyond 5G
}

\author{
Mimi Tam* \\ Department of Computer Science, University of Massachusetts, USA
}

*Corresponding author: Mimi Tam, Department of Computer Science, University of Massachusetts, USA.

Received Date: January 22, 2021

Published Date: January 25, 2021

\section{Introduction}

No question we are moving towards a "fully" digital and connected world. No question we are nowhere near the finish line and no question we shall get there one day. We knew it is within our ability to get there perhaps even in god speed.

5G monetization and adoption strategies had been elusive to CSPs (Communications Service Providers), MNOs (Mobile Network Operators), MVNOs (Mobile Virtual Network Operators), device manufacturers, network software vendors, systems integrators, incumbent Telecom/Wireless operators and all sorts of enterprises alike for some time, and new business models were said to have to be revamped to bring on real \& new revenues. When the $5 \mathrm{G}$ ecosystem emerges, the possibilities and opportunities for all parties involved are almost infinite although reaping revenues from these possibilities and opportunities may not all be immediate or even short term coming.

The promise of $5 G$ sounds marvelous \& works quite well in urban areas. As more and more $5 \mathrm{G}$ is available - Verizon $5 \mathrm{G}$ nationwide is available in $1,800+$ cities, AT\&T 5 G covers $15.99 \%$ of the United States, behind T-Mobile but ahead of Verizon's (as of Dec, 2020), a good number of $5 \mathrm{G}$ subscribers (consumer and business subscribers alike) may be a bit confused about what to expect in terms of its superior level of speed, latency \& reliability that $5 \mathrm{G}$ is supposed to offer.

By and large, there are 3 main network approaches in deploying 5G. The FWA (Fixed Wireless Access), Dynamic Spectrum Sharing and mmWave Ultra-Wideband network.

5G Fixed Wireless Access (FWA) employs standardized 3GPP architectures and common mobile components allowing network operators to deliver ultra-high-speed broadband services to suburban \& rural areas, supporting residential subscribers and enterprise customers where fiber is prohibitively expensive to lay and maintain. It features 5G New Radio (NR), 4G Evolved Packet Core (EPC) infrastructure for control and data transport and a new gNodeB (gNB) operating alongside an existing 4G eNodeB in the millimeter wavelength (mmWave). This is one of the so-called Non-Standalone (NSA) deployment options that can provide the bandwidth required to support high definition streaming services and high-speed Internet access; a viable alternative to fiber, cable and DSL.

This Non-Standalone (NSA) deployment option permits 5G NR and 4G LTE to coexist, allowing network operators an early rollout of $5 \mathrm{G}$ and a smooth transition from LTE to 5G NR. The NSA option uses LTE as the anchor to exchange signaling and control information. The anchor is there to handle (i.e. configure/modify/ add/delete/release) the connections to the 5G NR. The LTE eNodeB is the master cell group, the $5 \mathrm{G}$ gNB is the secondary cell group, and both RANs connect to the LTE core network.

Dynamic Spectrum Sharing (DSS) is an antenna technology that enables simultaneous use of $5 \mathrm{G}$ and LTE in the same frequency band. This technology can determine the demand for LTE and $5 \mathrm{G}$ in real-time and optimize the use of the airwaves by enabling multiple categories of users to safely share the same frequency bands.

If you have a new iPhone 12 with 5 G DSS service sitting at home in update New York first time downloading a 4 hour movie expecting a 1 minute turn-around time but it performed just as slow as your little brother's 4G/LTE Samsung Galaxy J3 phone, don't be surprised. Verizon never promised its 5G DSS gigabit speeds and 
sub-10-millisecond latency. 5G DSS can never deliver both as you might expect.

As a 5G DSS user with a 5G smartphone and you are situated within the radius of a DSS antenna but in a rural area, you are not going to get gigabit speed and sub-10-millisecond latency nor if you have a $4 \mathrm{G}$ phone with DSS service.

Millimeter wave (mmWave) is the short-range, high-frequency band of wavelength between $30 \mathrm{GHz}$ and $300 \mathrm{GHz}$ that will give you the gigabit speeds and sub-10-millisecond latency but limited range and no penetration to speak of, your office walls will block it and it needs clear line-of-sight. It only works in cities where inter-cell site distances are relatively small: up to $\sim 150$ meters, and it performs best when the sun is out as millimeter-wave signal strength will degrade somewhat when it rains.

This mmWave ultra-wideband network coverage that requires a lot of base stations everywhere in order to provide this ultrafast, ultra-reliable, ultra-low latency network in cities and in rural areas alike is not here today as of yet. However, performance and coverage will continue to grow over time.

\section{Today's 5G Can Power Business into Much Greater Profitability}

The key phrase of the time is "Cloud Native Strategy". Enterprises are going through their Digital Transformation phase; moving data center(s) to the cloud, transforming legacy assets to be cloud-native ledgers using all cloud resources namely cloud compute, cloud storage, network resource from the cloud. Connectivity to \& from the cloud is via $5 \mathrm{G}$ - fast, reliable, secure and ultra-low latency.

Usage of cloud platforms and utilities (e.g. IaaS / PaaS) is first and foremost cost effective, combined with $5 \mathrm{G}$ for business - a killer package for innovation and for bringing in new services/revenue to increase the bottom line.

Let's say you created an app on the GCP (Google Cloud Platform) at one of its 24 data centers and used Google Cloud Platform's multi-regional deployment mode to deploy your app. This allows you to place your app in a geographical location among the 24 different Google Cloud data center locations, the one that is closest to your visitors when called for, wherever they may be. This ensures low latency and blazing fast load times if you have $5 \mathrm{G}$ business connectivity.

If you decide to move your app from GCP to AWS (Amazon Web Services) and take advantage of Amazon's lower subscription fees with $\sim 109$ data centers around the globe, doing so is easy as long as your $5 \mathrm{G}$ business connectivity remains intact.

Practical experience has shown that most cloud-native architectures favor managed services; the potential risk of having to migrate off of them rarely outweighs the huge savings in time, effort, and operational risk of having the cloud provider manage the service, at scale and on your behalf. $5 \mathrm{G}$ is the connectivity ticket to success.

\section{Tomorrow's 5G is Coming along Big Time}

Look around you and you'll likely see numerous Internet-ofThings devices nearby such as smart phone, smart refrigerator, smart coffee pot, smart photo frame; look outside the window of your house and you may see smart plant-watering system, smart lighting, smart metering from your electric company's smart grid system; look further down the road and you may see a Tesla Autopilot swirling by followed by a Model 3 running V2X (Vehicleto-everything) protocols carried by $5 \mathrm{G}$ no doubt; since you are living in downtown Manhattan.

Ralph Wilson Stadium, largest stadium in New York is having an event. As many as 74,000 simultaneous connections or more could be going at the same time in the confines of this 500-600k square footage. $5 \mathrm{G}$ makes this kind of massive scale communications possible. More and more virtual reality/augmented reality apps running smoothly as in real reality and is happening, e-Health with the hope of remote surgery as tactile feedback is becoming possible; tactile Internet is on the horizon, high speed mobility such as in Bullet Trains, UAV (Unmanned Arial Vehicle), Industry 4.0, full autonomy and automation on factory floors, Smart Cities, Holographic Telepresence and fully autonomous driving; not even the sky is the limit. There is literally unlimited usage and unimaginable areas that $5 \mathrm{G}$ as a connectivity agent/carrier have yet to touch upon and support.

We are just trying to collect all the behavioral \& performance results from $5 \mathrm{G}$ deployments around the globe. That will help us determine the gaps of $5 \mathrm{G}$ and put proposals together for defining $6 \mathrm{G}$

The 'G's are perhaps just marketing terms. The industry and the standards committee people never stopped working on the 'G's. All these ' $G$ 's starting from $1 G$ the landline is an evolution path where continuous work has been done non-stop and improvements made non-interrupted in areas of architecture, speed, latency, security, configuration, storage, robustness and reliability.

\section{Beyond 5G}

We are seeing the emergence of more \& more $5 \mathrm{G}$ local networks deployed by different stakeholders to serve verticals' specific needs. These are new value chains and specific service providers known as micro operators (u0). They build and operate small cell communication infrastructures and offer local context related services and content in closed networks for its own customers, act as neutral host for MNO's customers or serve both. 
The beyond-5G trending highlights several on-going facts of life - from small number of dominant of MNOs to emergence of a large number of local network operators; from owning infrastructure to leasing network slices on-demand; from outdoor macro cell deployment to indoor small cell networks; from exclusivity in spectrum access rights to operation in shared spectrum bands; from sharing between an operator and an incumbent to interoperator spectrum sharing and from a small number of nationwide spectrum licenses to a large number of local spectrum licenses [1].

\section{Challenges \& Conclusion}

Around 50 billion IoT devices will be in use around the world by 2030 [2]. Each IoT device has one or more sensors. Integrating energy characteristics in designing wireless protocols vs. high deployment could impact the efficiency of low-power network operations.

To a large extend, $5 \mathrm{G}$ networks are disaggregated and virtualized RANs. The networking equipment does not require dedicated hardware. RAN and Core networks are all in the cloud / edge, virtual MAC and virtual PHY running on top of generic hardware. SDN/NFV is already becoming the prevailing architecture. 5G/6G should be all 'G's, all SON (Self Organizing Networks) to the true sense of the word.

For extreme multi-connectivity, exploration has already started on THz, VLC (Visible Light Communications), mmWave and sub-6 GHz links.

More variety of wireless infrastructures for network deployment, as a heterogeneous network will be the norm to support. We have massive MIMO LTE-Pro and 5G, outdoor small cell, metro core network, metro cell, outdoor DAS (Distributed Antenna System), indoor DAS, 4G/LTE, 5G DSS, 5G Ultra-Wideband, etc. One network, all RATs (Radio Access Technologies) need to be part of the equation.
Cloud distribution across networks with artificial intelligent solutions driven by different verticals. Whole system architecture is changing with base station densification, mobile edge computing, fog computing at devices, etc. These different components and layers could get complicated and require complex configuration and management tools to provision, configure, orchestrate and control all the various parts of the system. Architecture frameworks like ONAP (Open Network Automation Platform) are essential for abstracting software and hardware components from all architectural layers, vendors, providers and operators and function as a comprehensive platform for orchestration, management and automation of network and edge computing services for all parties involved.

There could be a litany of other challenges we overlooked. What about backhauling in remote areas? Should regulation in remote areas be handled differently? Who should pay for remote area networks \& backhauling links? How should UL (Uplink) heavy traffic be handled? How does extremely long-range communication work? How does extremely short-range communications work? There will be so many more questions as we uncover the shortcomings of $5 \mathrm{G}$ when $5 \mathrm{G}$ gets more airtime \& mileage.

As a result, totally new $6 \mathrm{G}$ business ecosystem will likely emerge as the next ' $G$ ' generation kicks in.

\section{Acknowledgement}

None.

\section{Conflict of Interest}

No conflict of interest.

\section{References}

1. Holst A (2021) IoT connected devices worldwide 2030.

2. $2^{\text {nd }} 6 \mathrm{G}$ Wireless Summit 2020 proceedings (2020) Technical CoSponsorship by IEEE. 